\title{
Shape constancy and a perceptual bias towards symmetry
}

\author{
MICHAEL KING, GLENN E. MEYER, JOHN TANGNEY, and IRVING BIEDERMAN \\ State University of New York at Buffalo, Buffalo, New York 14226
}

\begin{abstract}
In attempting to judge whether a briefly presented rotated figure was symmetrical (circle or square) or asymmetrical (ellipse or rectangle), a bias towards perceiving symmetry was evident. This bias appears to be perceptual rather than judgmental in origin in that it was affected neither by variations in the probability of occurrence of symmetrical figures nor by familiarization with the stimuli. A characterization of the stimulus similarity space based on the shape-slant invariance hypothesis is presented, and a modtel which incorporates both the similarity space and the symmetry bias is proposed. The model can account for our paradoxical finding that the more asymmetrical some projected images, the more likely they will be perceived as symmetrical. This model can also account for Massaro's $(1973,1975)$ results on shape constancy. However, Massaro's theory was not supported in that extension in depth did not account for increased difficulty of shape judgments.
\end{abstract}

Through the operation of shape constancy, the true shape of an object tends to be perceived independently of the orientation of the object to the viewer's line of sight. The classical explanation of shape constancy phenomena is the shape-slant invariance hypothesis of Koftka (1935). Under the invariance hypothesis, the values of the projected shape of the object on the retina and the perceived orientation or slant of the figure uniquely determined the perceived shape of the object.

Many researchers have found that shape constancy declines as the angle of orientation increases (Epstein \& Park, 1963; Massaro, 1973; Stravrianos, 1945; Thouless. 1931). If there is an "invariant" relationship between slant and apparent shape, why does the degree of slant significantly affect the perceived shape of an object? This decrease in constancy at high angles of orientation could be accounted for in three ways: (1) the registration of the slant of the object could increase in difficulty as tilt increases, (2) the projected image of the object could be more difficult to process, or (3) the perceived shape, the product of the equation, could be influenced by some other variable, thus making this decision more difficult and time consuming. Massaro (1973) sought to systematically test each of these possibilities. Taking reaction time and errors as indices of difficulty, he tested slant discrimination, projected shape discrimination, and true shape judgment, each over varying angles of inclination. According to his processing time analysis of the invariance hypothesis, the times for slant and

This research was supported, in part, by National Institute of Mental Health Grant MH 22383 to Irving Biederman. The authors express their appreciation to D. W. Massaro and V. R. Carlson for their critical and helpful comments on earlier versions of this manuscript. Michael King is now at Duke University. Requests for reprints should be sent to Irving Biederman, Department of Psychology, State University of New York at Buffalo, 4230 Ridge Lea Road, Buffalo, New York 14226. projected shape judgments should affect the time needed for perceived shape judgments at all inclinations. Massaro found no corresponding increase in RTs of judgments of the slants of pairs of figures presented at increasing angles of orientation (Experiment IV). The increased similarity of projected shapes as angle of orientation increased led to only a relatively small increase in RTs and errors for same-different judgments of these shapes when presented in the frontal-parallel plane (FPP). This similarity effect was inferred to be too small to account for the increases in times noted for the perceived same-different shape judgments at increased angles of orientation (Experiment V) (though, in a later discussion, Massaro, 1975, allowed for a larger effect of similarity at extreme rotations).

Massaro (1973, 1975) proposed a perceptual encoding model of the real shape judgment process which attributed the effects of object rotation on reaction times to the time required for the viewer to fuse the two retinal inputs into one picture. Thus, according to the model, the critical independent variable is not the angle of orientation of a figure, but rather the distance between the fixated and farthest points of the test figures. If this distance exceeds Panum's area of binocular fusion, i.e., if the stimulus is large enough so that at a given angle of orientation its edges will fall beyond the $6^{\prime}-10^{\prime}$ minutes of visual angle which define Panum's area, then vergence movements to provide a clear (fused) image for accurate shape registration will be required. ${ }^{1}$ According to Massaro's model, it is the time for these vergence movements which results in increased $R T$ s at the more extreme angles of orientation. For a given angle of orientation, the more a stimulus exceeds Panum's area, the greater the time required for fusion to occur.

In what he termed a critical test of this theory, Massaro (1975) studied the effects of rotation on the speed of same-different shape judgments of pairs of 
stimuli that were reduced in width (and had an extension in depth when rotated that did not exceed Panum's area) compared to those of the original (1973) Experiment V. The RTs for shape judgments of these narrower stimuli were only slightly lengthened by rotation, whereas those RTs in the original (1973, Experiment V) study showed a much larger effect of rotation. Massaro (1975) interpreted this result as supporting his extension in depth hypothesis over the shape-slant invariance hypothesis. ${ }^{2}$ However, when Massaro narrowed his figures, he kept the height constant. Consequently, the values of the height-towid th ratios of the figures were not exactly comparable in the two studies. Thus, in the original (1973) experiment, the height-to-wid th ratios were highly similar (1 and 1.18), while in the 1975 experiment, the figures were more dissimilar (ratios of 2.32 and 3.00). Depending on how discrimination was affected by different height-to-width ratios, the lack of an effect of rotation in the 1975 study could have been a consequence of the greater dissimilarity in the shapes of the to-bediscriminated figures (larger differences in height-towid th ratios) making for an easier discrimination-one that would be less susceptible to rotation. If such an explanation can be shown to have merit, then Massaro's results could be readily accommodated by the shape-slant invariance hypothesis.

The present study was designed to independently vary the width (and therefore extension in depth) and similarity of the height-to-width ratios of the comparison figures. This would allow an unconfounded test of Massaro's hypothesis that extension in depth of a figure rotated away from the FPP is critical. Whatever the ultimate resolution of the effects of rotation on shape constancy, Experiment I can be regarded as providing an assessment of the relative effects of extension in depth and similarity on shape constancy. Perhaps of even greater interest was the appearance of a strong bias favoring symmetrical (vs. asymmetrical) judgments in Experiment 1 . Experiments II and IIIwere designed to explore the nature of this bias.

In the present investigation, subjects viewed circles and ellipses, or squares and rectangles. Each shape came in three different sizes, and was presented at three different angles of orientation and for three different durations. Subjects were to make absolute shape judgments as to whether a single figure was a circle or an ellipse, or, in the other condition, a square or a rectangle. The large figures should, if Massaro's perceptual encoding model is correct, be mistaken more often than the small figures. Increased durations should reduce the effect of size. The square-rectangle discrimination was included to determine if shape constancy operates in a similar manner across differences in shape. A rating scale was used in an attempt to further assess perceptual difficulty and response bias.

\section{EXPERIMENT I}

\section{Method}

Subjects. Six male and four female graduate and undergraduate student volunteers, aged 19-25, served as subjects.

Procedure. Each subject was tested once in one 45-min session consisting of two blocks of trials. A block consisted of 54 presentations of either a circle-ellipse series or a square-rectangle series. Half the subjects received the circle-ellipse block first and the other five subjects the square-rectangle block first. The order of conditions within a block was randomly and separately determined. Thus, for a circle-ellipse series, subjects saw three circles and three ellipses, each object for three different lengths of time and at three different angles of inclination and at all possible combinations of conditions for a total of 54 presentations. After a short break, subjects would view the second block of trials for a total of 108 trials per subject.

The subjects were instructed as to the general notion of shape constancy, using the test stimulus as an example. It was shown that a circle tilted away from FPP could be regarded as an ellipse, but it was stressed to the subjects that the experimenter was interested in the real shape of the objects they were about to see. Subjects were told they were going to be shown a series of objects of different sizes (either circles-ellipses or squares-rectangles) at different angles to their line of sight and for different lengths of time. It was again stressed that the experimenter was interested in the real shape of the object. The workings of the response sheet and rating scale and the shutter trigger were explained to the subjects and the test object installed in the box. All subjects saw the test object at an orientation of $39^{\circ}$ for $100 \mathrm{msec}$. They were allowed to release the shutter as many times as they wished until they were familiar with its workings. The shutter was operated by the subjects once per experimental trial. They were told of the random nature of the experimental conditions and were advised not to try to anticipate the next trial. No feedback was given as to response accuracy. The objects were changed by the experimenter while the subject was recording his response and rating. During this interval, the experimenter also changed the time setting on the shutter by pulling back the projector and adjusting the shutter dial. The object was removed and replaced whether required by the random arrangement or not. The time device was also adjusted whether required or not.

Stimuli. Test stimuli consisted of 12 flat objects, all cut from the same $.2-\mathrm{cm}$ heavy white paperboard. There were three each of circles, ellipses, squares, and rectangles. Dimensions ${ }^{3}$ of the stimuli were as follows: circles with diameters of $5.1,4.3$, and $2.2 \mathrm{~cm}$; ellipses with major vertical axes of $5.1,4.3$, and $2.2 \mathrm{~cm}$, and with minor, horizontal axes of $4.3,2.2$, and $1.7 \mathrm{~cm}$, respectively; squares and rectangles had the same dimensions as the circles and ellipses, respectively. All the figures were painted a uniform silver which minimized textural depth cues. Since the figures were not perfectly two-dimensional, the back edges of the forward side of the circles and ellipses were gound to eliminate curvature cues to the object's shape. Thus, upon rotation of the figures, no edge width was apparent. This was necessary as a comparison between the perception of circles-ellipses and squares-rectangles was sought. The straight line edges of the squares and rectangles presented no clues to the object's shape. In contrast, the varying degree of the side curvature of the circles-ellipses gave information as to the object's shape across different sizes. The figures were glued to the wire standards. The long axis of the ellipses and rectangles was vertical. A single practice stimulus consisted of a $7.6-\mathrm{cm}$ circle cut from the same material and painted bright red.

Apparatus. The apparatus consisted of a display box, a projector as light source, and a variable speed shutter. The box was constructed of $.9 . \mathrm{cm}$ plywood and was $87.7 \times 53 \times 70.4 \mathrm{~cm}$. The floor of the box rested on a tabletop. The figures were held in place within the box by a $1.9-\mathrm{cm}$ mount board secured to the floor of the box. A $5.4-\mathrm{cm}$ hole was drilled in the center of this mount board, 
allowing easy placement and removal of stimulus objects while maintaining exact position. Angular measurements of $0^{\circ}, 26^{\circ}$, and $65^{\circ}$ relative to the FPP plane of the box were marked on the mount board. $0^{\circ}$ equalled the FPP of the object. The mount board was centered horizontally in the box and was $19.6 \mathrm{~cm}$ from the back wall.

Each stimulus object was centrally mounted on a heavy steel wire, $3 \mathrm{~mm}$ thick and $34.9 \mathrm{~cm}$ high. The wire stand ards were secured in $5.1 \mathrm{~cm}$ circular wooden mounts, $1.9 \mathrm{~cm}$ thick. A marking on the mount designated the point exactly $90^{\circ}$ to the plane of the stimulus object. This marking, in conjunction with the angle designations on the mount board. allowed the stimulus objects to be presented at known angles to the viewer's line of sight. Rotation was around the vertical axis with the furthest edge to the viewer's right. Stimulus objects were changed through a small hinged door, $22.4 \mathrm{~cm}$ high, in the bottom of the rear wall. A partition standing $28 \mathrm{~cm}$ high was placed across the box $51.5 \mathrm{~cm}$ from the front wall. This partition occluded the door in the back wall and all but $6.4 \mathrm{~cm}$ of the stimulus standards. The entire inside of the box and the stimulus standards were painted black. The stimuli were viewed binocularly through a rectangular slit, $12.7 \times 4.6 \mathrm{~cm}$, in the front wall on a point $35.1 \mathrm{~cm}$ from the bottom of the box and $26.4 \mathrm{~cm}$ from either side. An extended viewer limited any large head movements of the subject. The distance between the stimulus and the viewer's eyes was $73 \mathrm{~cm}$.

The light source was a Kodak Carousel AV-900 projector fitted with an Ilex No. 3 Universal variable speed shutter. The shutter allowed manual variation in duration of light flashes. Presentation times were 40,200 , and $500 \mathrm{msec}$. The luminance of the figures was $29 \mathrm{~cd} / \mathrm{m}^{2}$. The shutter was triggered by a manual plunger device attached to the shutter by a flexible cord. This trigger device was subject-operated throughout the experiment. Both the projector and shutter were situated outside the box and rested on the table at the side of the box. Light from the projector entered the box through a $5-\mathrm{cm}$ hole drilled in the left side of the box at the lower right-hand corner. A nozzle device fit snugly against the surface of the shutter eliminating any extraneous light from entering the box. A mirror located inside the box and angled across and up from the corner opposite the projector effectively reflected the light flashes onto the stimulus objects without shadows or other distracting effects.

Ratings. The subject entered his judgment and confidence rating (5.point scale from 1 "not confident" to 5 "very confident") on a response sheet. Numbered spaces were provided for each of the 54 trials per block for both blocks. Thus, for each experimental trial a response was obtained as to the viewer's determination of the shape of the object along with a subjective rating as to the subject's confidence in the correctness of his response.

\section{Results}

The primary data were errors in judgments as to the shapes of the figures. These data were analyzed by a 2 by 2 by 3 by 3 by 3 factorial design with $\mathrm{n}=10$. The variables were shape (circular vs. angular), symmetry (circle-square vs. ellipse-rectangle), size (small, medium, large), angle of orientation $\left(0^{\circ}, 26^{\circ}, 65^{\circ}\right)$, and viewing time $(40$, 200 . and $500 \mathrm{msec}$ ). Signal detection analyses were performed on the rating scale data. These yielded the same results as the error data and will not be discussed further.

The data with the round stimuli (circles and ellipses) were only negligibly different from the data with angular (squares and rectangles) stimuli. Figure 1 show's the data for the ellipses combined with the rectangles (asymmetrical figures) and the circles combined with the squares (symmetrical figures).

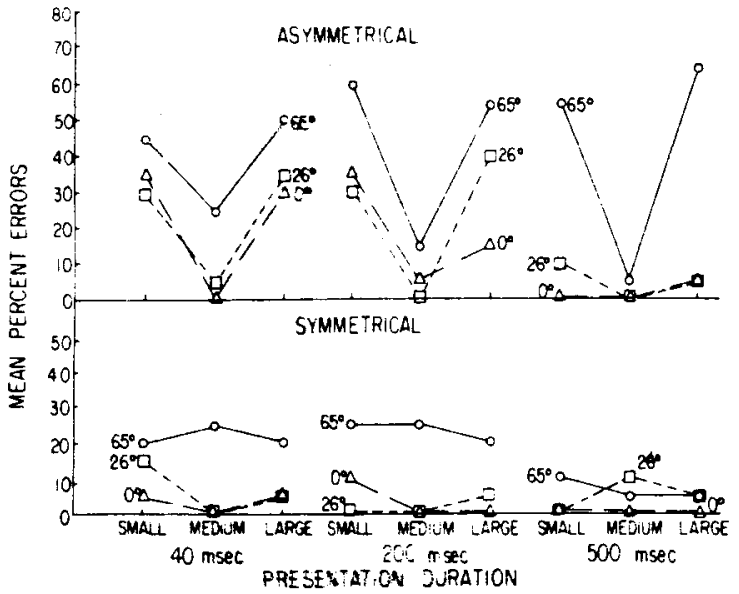

Figure 1. Mean percent errors in Experiment $I$ as a function of symmetry, presentation duration, stimulus size, and angle of orientation.

Overall, both presentation duration and angle of orientation led to expected main effects. The 40- and $200-\mathrm{msec}$ presentation durations yielded very similar error rates of $19.4 \%$ and $18.9 \%$, respectively. However, objects viewed for $500 \mathrm{msec}$ were judged more accurately, with an error rate of only $10.5 \%$; $\mathrm{F}(2,18)=14.28, \mathrm{p}<.001, \mathrm{MSe}=.071$. The overall error rates for objects at $0^{\circ}, 26^{\circ}$, and $65^{\circ}$ angles of rotation were $8.1 \%, 10.8 \%$, and $29.4 \%$, respectively, $\mathrm{F}(2,18)=41.48, \mathrm{p}<.001, \mathrm{MSe}=.117$.

Contrary to expectations from Massaro's (1973. 1975) extension in depth theory, the large stimuli did not result in a noticeably higher error rate than the small stimuli $(21.4 \%$ vs. $20.3 \%$, respectively). But what is clearly embarrassing to Massaro's theory was the absence of a greater effect of angle of rotation on the large stimuli compared to the small stimuli. (The large stimuli would be more likely to fall outside of Panum's area when rotated.) If anything, the opposite was true. As the angle of orientation increased from $0^{\circ}$ to $65^{\circ}$, the error rates for the small stimuli increased $26.6 \%$ while the corresponding increase for the large stimuli was $21.6 \%$. Overall, though, the Size by Angle interaction was simply not significant, $\mathrm{F}(4,36<1.00$, MSe $=.107$. Massaro's theory also implies that, at extreme angles of rotation, the large stimuli would derive a greater benefit from increased presentation duration since more time would be available for vergence movements. This did not occur. In fact, at the $65^{\circ}$ angle of orientation, increasing presentation duration from 40 to $500 \mathrm{msec}$ did not improve accuracy for either the small or the large stimuli.

Subjects in this task had to choose some criterion value of symmetry in order to respond. If the height-to-width ratio was around 1.00, the subject could respond circle or square; otherwise, he would respond ellipse or rectangle. The similarity in height-to-width ratio between an asymmetrical figure and its symmetrical counterpart (in size and 
roundness-angularity) would be one determinant of accuracy: the more similar the height to width ratios, the more difficult the task. In the present experiment, the medium-sized asymmetrical figures were of a height-to-width ratio of 1.95 . This value represents a considerably greater departure from symmetry than did the height-to-width ratios of the small and large asymmetrical figures, which were 1.29 and 1.19 , respectively. Consequently, other things being equal, the medium-sized figures should be easier to identify than the small or large figures. The latter figures, because of their highly similar height-to-width ratio, were of approximately equal identifiability. The low error rates for the asymmetrical medium-sized stimuli produced a significant effect of size, $F(2,18)=17.72$, $\mathrm{p}<.001$. MSe $=.136$, and a significant Size by Symmetry interaction, $F(2,18)=7.30, p<.01$, MSe $=.282$. It must be emphasized that these effects of stimulus size are completely attributable to the reduced symmetrical-asymmetrical similarity of shape of the asymmetrical medium stimuli. When similarity was held constant. as with the small and large stimuli, no independent effect of size was apparent in these data.

In the introduction, we offered an explanation of Massaro's (1975) result of smaller effects of rotation with narrower figures. According to this alternative explanation, the greater dissimilarity of the shapes of these figures could have rendered them less susceptible to rotation. The results of Experiment I support this explanation: The medium asymmetrical stimuli, which were most dissimilar to the symmetrical stimuli, showed an increase in error rate of only $13.3 \%$ when rotated from $0^{\circ}$ to $65^{\circ}$, whereas the small and large asymmetrical stimuli, which were more similar to the symmetrical stimuli, showed an increase in error rate of $35.0 \%$ as they were rotated from $0^{\circ}$ to $65^{\circ}$.

The most striking feature of the data shown in Figure 1 is the effect of symmetry. Asymmetrical figures led to considerably higher error rates $(24 \%)$ than did the symmetrical figures $(8 \%), F(1,9)=$ 16.00. $\mathrm{p}<.01$. MSe $=.4481$. There was a clear bias toward responding with the symmetrical response. Though symmetrical and asymmetrical stimuli were presented with equal frequency, the symmetrical response (i.e., circle or square) was made on $58 \%$ of the trials.

But what is remarkable is the contrast in the effects of rotation for the symmetrical and asymmetrical figures. The increase in error rates for the symmetrical figures at increased angles of rotation is in the expected direction with respect to projective shape: as the projected shape of the symmetrical figures becomes more and more asymmetrical, the proportion of (erroneous) asymmetrical judgments increases. But, the increase in error rates for the asymmetrical figures at increased angles of rotation is in a direction opposite to what would be expected on the basis of projective shape. That is, for the asymmetrical figures, as their projective shapes become more asymmetrical, there was increased tendency to (erroneously) judge them to be symmetrical!

While there was a consistent decrease in errors of rotated symmetrical figures with increased presentation duration. such a decrease did not consistently occur with the small and large asymmetrical figures when rotated to $65^{\circ}$. In fact, the error rates for the small and large asymmetrical figures fell farther below chance accuracy as presentation duration was increased from $40 \mathrm{msec}$.

\section{Discussion}

What model might explain these and Massaro's (1973) data? While Massaro's objective of describing the information processing implied by the shape-slant invariance hypothesis is desirable, we believe that he erred in rejecting that hypothesis. It will be remembered that a critical point in Massaro's argument against the invariance hypothesis was that RTs and errors for slant judgments did not increase with increased rotation. Massaro reasoned that the reduced shape discriminability at increased angles of rotation could not be explained by poorer slant discrimination at these angles of rotation. While this conclusion is true, it does not follow that the increase in RT for judging true shapes with increases in rotation cannot be attributed to the increased slant. Essentially, what Massaro demonstrated in his Experiment IV was that the resolution of rotation discrimination did not change with angle of rotation. But, as symmetrical and asymmetrical figures are rotated, the height-to-width ratio of their projections become more similar. Therefore, the tolerance for their discrimination given some (constant) error of resolution of slant or height-to-width ratio is reduced as rotation increases.

The results from Massaro's $(1973,1975)$ and our own experiments can be understood by consideration of three factors: (1) The subject's task is one of discriminating symmetrical from asymmetrical figures; (2) as the stimuli are rotated, their projected width-to-height ratios ${ }^{4}$ become increasingly similar; (3) when projected width-to-height ratios are similar, i.e., when discrimination is difficult, a bias which favors judgments of symmetry can manifest itself.

These relations can be appreciated by an examination of Figure 2, which shows the projected width-to-height ratio of the symmetrical and asymmetrical stimuli as a function of the angle of rotation (orientation). It is clear that as the stimuli in the present experiment were rotated, the differences in the projected width-to-height ratio were reduced. Since the subject's task of discriminating symmetrical from asymmetrical stimuli can be regarded under the 


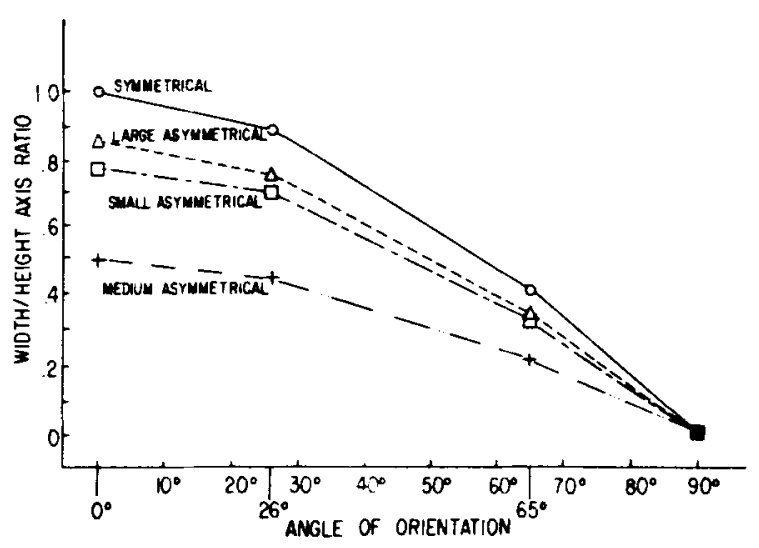

Figure 2. Projected width-to-height ratio as a function of orientation stimulus symmetry, and size.

invariance hypotheses as one of judging objective width-to-height ratio on the basis of projected width-to-height ratio and angle of orientation, Figure 2 can be interpreted as a model of the confusability space for discriminating symmetrical from asymmetrical stimuli. Given some opportunity for error in judging either the projected width-to-height ratio (an error of judgment of the projected shape of the stimulus) or in judging the angle of orientation, the closer an asymmetrical stimulus is to the corresponding symmetrical stimulus, the more likely will either of the two kinds of errors result in an error in the final symmetricasymmetric judgment. Since the width-to-height ratio of the symmetrical and asymmetrical figures become more similar with rotation, any constant error of judging angle of rotation or shape (width-to-height ratio) will cause increasing errors as the angle of rotation increases. For example, if the large asymmetrical stimulus were presented at $0^{\circ}$ and its width-to-height ratio accurately judged, then even an $8^{\circ}$ error in judging its angle of orientation would still not result in the large asymmetrical stimulus being confused with its symmetrical counterpart, since they are separated by $31^{\circ}$-i.e., the symmetrical and large asymmetrical stimuli at $0^{\circ}$ are horizontally separated by $31^{\circ}$ - the symmetrical stimulus would have to be rotated to $31^{\circ}$ to have a projected width-to-height ratio of .84 , the width-to-height ratio of the large asymmetrical stimulus. However, if the large asymmetrical stimulus was presented at $65^{\circ}$, then an $8^{\circ}$ error in judging its angle of orientation could readily result in its being confused with the symmetrical stimulus. An error in judging the orientation of the large asymmetrical stimulus at $65^{\circ}$ of only $+5^{\circ}$, along with an accurate judgment of the width-to-height ratio of the figure, yields, according to Figure 2 and the invariance algorithm, the same projected shape as the large symmetrical figure.

A parallel argument can be constructed for errors in judging the projected width-to-height ratio.
Tolerance for these errors are reflected by the vertical distance between the symmetrical and asymmetrical functions. Just as the tolerance of judgments for errors of slant judgment is reduced as angle of orientation is increased, the tolerance for errors in projected width-to-height ratio judgments is reduced as the angle of orientation is increased. That is, the vertical distances between symmetrical and a given asymmetrical function is reduced as the angle of orientation is increased.

Figure 2 suggests that at small angles of rotation, most errors would be produced by errors in the judgment of projected width-to-height ratios. The relative contribution of slant judgment errors would increase with increased angles of orientation. We have not extensively explored models for the combined effects of the two sources of errors. However, simple city block distance functions (Shepard, 1964), in which the horizontal and vertical distances between the symmetrical functions and a given asymmetrical function are summed, yielded a reasonably good fit to the error data. For the nine conditions (three circle sizes times three angles of rotation) at 40,200 , $500 \mathrm{msec}$, the respective correlations were $-.91,-.80$, and $-.65(\mathrm{df}=8, \mathrm{p}<.01, .01$, and .05 , respectively) between percent errors on the asymmetrical stimuli and the sum of the horizontal and vertical distances between the asymmetrical points in Figure 2 and the symmetrical function.

Given that a stimulus is close enough to be confused with its symmetrical or asymmetrical counterpart, the advantage of the symmetrical stimuli suggests that there is a bias for classifying stimuli as symmetrical. In terms of Figure 2, confusion between a given asymmetrical function and the symmetrical functions are much more likely to go from left to right or from bottom to top than they are to go in the opposite directions.

We are not sure why increased presentation durations result in more errors on the (difficult) small and large asymmetrical stimuli at $65^{\circ}$ angle of orientation. This effect (which was also found in Experiment II) could occur if there was some tendency to underestimate extreme angles of orientation at brief presentation durations. With increased duration, the angle of orientation would be more accurately judged - that is, it would be judged to be larger than it was at the briefer presentation durations. If the subject judges the angle of orientation to be larger, then he is required to discriminate between asymmetrical and symmetrical projected width-to-height ratios that are much more similar (as shown in Figure 2). The more similar the ratios, i.e., the more similar the projected shapes of symmetrical and asymmetrical pairs, the greater the confusability. Since confusions tend to be resolved with a bias toward symmetry, the more accurate judgment of the extreme slant of asymmetrical stimuli 
increases the likelihood of these stimuli being judged as symmetrical.

The following example should clarify the preceding explanation. Assume that when the large asymmetrical ellipse was presented for $20 \mathrm{msec}$ at $65^{\circ}$, its width-to-height ratio was accurately perceived at .36 (see Figure 2) but that its slant was underestimated at $26^{\circ}$. A circle presented at $26^{\circ}$ would have a projected width-to-height ratio of 90 . This is so discrepant from the .36 width-to-height ratio of the ellipse that the subject rarely confuses the two. At $500-\mathrm{msec}$ presentation durations, assume that the subject accurately perceives the slant angle at $65^{\circ}$. But now a circle at $65^{\circ}$ would have a projected width-to-height ratio of .43 , which is so similar to the .36 width-to-height ratio of the ellipse that he frequently confuses the two (with a bias towards symmetry).

How should the symmetry bias be characterized? It is tempting to regard it as a simple response bias in which the subject tends to select the symmetrical response when uncertain as to the correct choice. But such a characterization does injustice to the wide variety of phenomena (e.g., the Ames room and the trapezoidal window) in which the bias toward symmetry appears to exert genuine perceptual effects. Experiment II was designed to investigate the nature of the symmetry bias.

\section{EXPERIMENT II}

Experiment II investigated the effects of variations in the probability of occurrence of symmetrical vs. asymmetrical stimuli. A positive effect of the probability variable would be equivocal in deciding between a perceptual vs. response bias interpretation of the symmetry effect. If performance can be demonstrated to be independent of probability, then a perceptual effect would be suggested.

If the symmetry bias revealed in Experiment $I$ was a response bias, variations in the probability of occurrence of the symmetrical and asymmetrical stimuli should affect the tendency to use one or the other response category. Such a probability effect could also be interpreted as a perceptual effect if it is assumed that probability affects perceptual encoding. Since response biases are typically interpreted to be under voluntary control, a failure to find an effect of probability would suggest a perceptual basis of the symmetry bias: knowing that the trapazoidal window or the Ames room is not symmetrical does not eliminate these illusions.

\section{Method}

Save for the following exceptions, the method and procedure of Experiment II was identical to that of Experiment I. The subjects were 14 male and 6 female undergraduates, aged $17-20$, recruited from the undergraduate psychology subject pool. Whereas in Experiment I there were equal numbers of stimuli in each of two blocks, in Experiment II each of four groups of five subjects had one block of a given stimulus probability and shape condition. Each group received one of the four possible combinations of $1 / 3$ symmetrical (circles or squares) and $2 / 3$ asymmetrical (ellipses or rectangles) figures or $2 / 3$ symmetrical and $1 / 3$ asymmetrical figures. This was done within each group by replicating a given series of 27 presentations of 3 durations by 3 sizes by 3 angles of rotation for either the $2 / 3$ symmetrical (either circles or squares) or the $2 / 3$ asymmetrical (either ellipses or rectangles) stimuli. This change resulted in a decrease in presentations from 108 (Experiment I) to 81 stimuli (Experiment II). It will be recalled that the small and large asymmetrical stimuli in Experiment I did not have identical height-to-width ratios. This was corrected by reducing the width of the large stimuli from 4.3 to $3.94 \mathrm{~cm}$ to yield a height-to-width ratio of 1.29 , which was identical to the small stimuli. Before each block, subjects were fully instructed as to the relative likelihood of occurrence of circles to ovals or squares to rectangles. The rating scale was changed to one which went from 1 (most confident symmetric) to 10 (most confident asymmetric).

\section{Results}

There was no effect of the probability manipulation on the symmetry bias. Overall, symmetrical stimuli were incorrectly identified on $12.4 \%$ of the trials compared to $20.3 \%$ for the asymmetrical stimuli. For the group with $1 / 3$ symmetrical stimuli, the error rates were $11.8 \%$ and $22.4 \%$ for the symmetrical and asymmetrical stimuli, respectively. For the group with $2 / 3$ symmetrical stimuli, the error rates were $13.0 \%$ and $18.1 \%$ for the symmetrical and asymmetrical stimuli, respectively. If anything, the direction of this negligible effect is opposite to what would be expected from the probability manipulation: increasing the frequency of symmetrical stimuli increased the error rates to those stimuli and reduced the error rate to the asymmetrical stimuli.

Experiment II closely replicated the results of Experiment 1. Again, the confidence data was virtually identical to the accuracy data. As in Experiment I, stimulus shape (angular vs. curved) had no effect. A 2 (probability of symmetrical stimuli) by 3 (duration) by 3 (size) by 3 (angle of orientation) by 2 (symmetry) analysis of variance was performed on the mean error rates. Neither the main effect of probability nor the Probability by Symmetry interaction approached significance $(F<1.00$ in both cases). Figure 3 shows the results for the other major experimental variables. Presentations durations of 40 , 200 , and $500 \mathrm{msec}$ resulted in error rates of $20 \%$, $16 \%$, and $13 \%$, respectively, $F(2,36)=5.43$, $\mathrm{p}<.01, \mathrm{MSe}=.083$. Angles of orientation of $0^{\circ}$, $26^{\circ}$, and $65^{\circ}$, yielded error rates of $6 \%, 10 \%$, and $33.7 \%$, respectively, $F(2,36)=59.30, \mathrm{p}<.001$, MSe $=.134$. The effect of symmetry described above was also significant: $\mathrm{F}(1,18)=6.29, \mathrm{p}<.05, \mathrm{MSe}=.266$.

The small, medium, and large figures had error rates of $21.2 \%, 12.2 \%$, and $15.6 \%$, respectively, $F(2.36)=10.63, p<.001, \mathrm{MSe}=.750$. The higher error rates on the small stimuli, relative to the large stimuli, is opposite to what would be expected from Massaro's theory. As the angle of rotation increased from $0^{\circ}$ to $65^{\circ}$, error rates on the small stimuli in- 


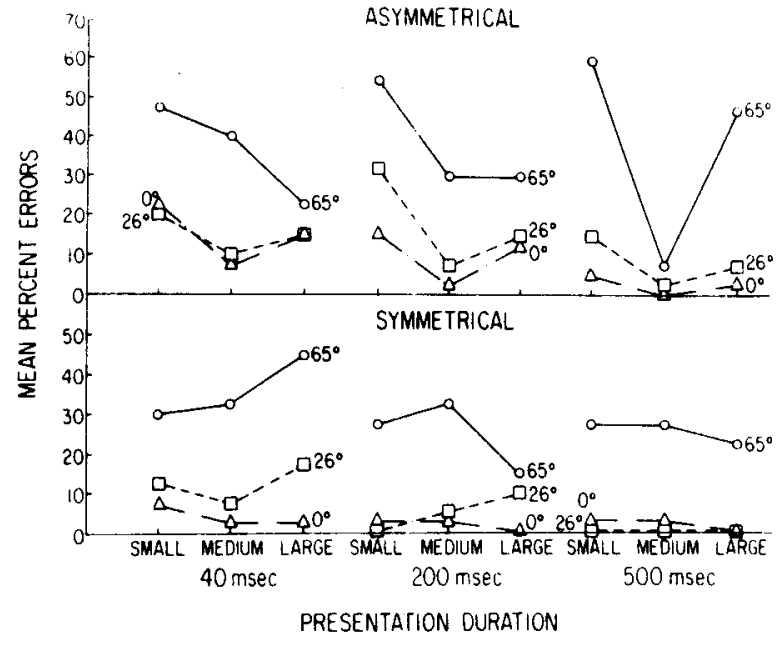

Figure 3. Mean percent errors in Experiment II as a function of symmetry, presentation duration, stimulus size, and angle of orientation.

creased $32 \%$ while the corresponding increase for the large stimuli was $25 \%$. At $65^{\circ}$, increased presentation duration from 40 to $500 \mathrm{msec}$ did not noticeably reduce the error rate for the large stimuli relative to the small stimuli. In fact, the error rate increased by $1.2 \%$ for the large stimuli and by $5.0 \%$ for the small stimuli. The lack of a beneficial effect of increased presentation duration at this extreme angle of rotation in both Experiments Iand II casts additional doubt as to the role of vergence movements in accounting for the effect of angle of orientation.

The only interaction that was significant was that of Size by Symmetry, $F(2,36)=8.50, \mathrm{p}<.01, \mathrm{MSe}=$ .061 . The symmetry effect was primarily evidenced by the small and large stimuli. As in Experiment $I$, the asymmetrical stimuli which were least similar to the symmetrical stimuli (i.e., the medium figures) showed a smaller effect of rotation compared to those asymmetrical stimuli which were most similar to the symmetrical stimuli (i.e., the small and large stimuli). The error rate on the medium asymmetrical stimuli increased by $22.5 \%$ as the angle of rotation increased from $0^{\circ}$ to $65^{\circ}$ compared to a $31.6 \%$ increase in this error rate for the small and large asymmetrical figures. The comparisons of these effects for the small and large asymmetrical stimuli prove to be damaging to Massaro's model: The increase in error rate with rotation for the small stimuli was $40.0 \%$, which is more than the $23.3 \%$ increase for the large stimuli.

The correlations between error rates on the asymmetrical stimuli and the sum of the city block distance between the symmetrical and asymmetrical functions in Figure 2 yielded correlations of -.70 , -.83 , and $-.73, \mathrm{p}<.05, .01$, and $.05(\mathrm{df}=8,3$ sizes by 3 angles of rotation) for the 40-, 200-, and 500-msec presentation durations, respectively.

\section{EXPERIMENT III}

It could, of course, be argued that our procedures in Experiment II were somehow inadequate for demonstrating an effect of probability on the symmetry bias. However, other work in our laboratory, using similar instructional procedures (Biederman \& Stacy, 1974; Biederman \& Zachary, 1970), has succeeded in demonstrating stimulus probability effects on reaction time for much/smaller probability differences (e.g., probability values of .067 vs. .2) even when both stimuli were assigned to the same response so that an overt response bias component was eliminated.

The obligatory - and, by present reasoning, the perceptual-nature of the symmetry bias was further underscored by the results from five additional subjects, four males and one female, aged 19-22, who were each run on a circle-ellipse discrimination as Experiment III. Two of these subjects were run with a block with $1 / 3$ circles and $2 / 3$ ellipses; the other three subjects were run with $2 / 3$ circles and $1 / 3$ ellipses. The method and procedures of Experiment II were followed, with the sole difference being that these five subjects were given an opportunity to closely examine and compare stimuli in the experiment. The six stimuli (three sizes of circles and ellipses) were displayed on a table for the subject to study prior to the experiment.

\section{Results}

Given but slight allowance for increased variability due to the small number of subjects, the data from these five subjects were quite similar to the data from Experiments I and II. The overall error rate was $17 \%$ (compared to $16 \%$ in Experiments I and II), so the increased familiarization did not increase accuracy. Moreover, the symmetry bias was still present. Overall, circles were incorrectly responded to on $14 \%$ of the trials compared to an error rate of $22 \%$ when ellipses were presented. This $8 \%$ advantage for the circles was identical to what was obtained in Experiment II (but smaller than the $16 \%$ difference in Experiment I). As in Experiment II, the probability manipulation failed to affect judgments in the expected direction. The three subjects with $1 / 3$ symmetrical stimuli had error rates of $25 \%$ and $11 \%$ on ellipses and circles, respectively. However, the two subjects with $2 / 3$ symmetrical stimuli yielded error rates of $17 \%$ and $15 \%$ on ellipses and circles, respectively. Other aspects of the data were quite similar to those of Experiments I and II. Small, medium, and large stimuli yielded error rates of $27 \%$, $11 \%$, and $18 \%$, respectively. Angles of rotation of $0^{\circ}$, $26^{\circ}$, and $65^{\circ}$ yielded error rates of $4 \%, 8 \%$, and $44 \%$, respectively. 


\section{CONCLUSIONS}

The invariance hypothesis, coupled with a perceptual bias toward symmetry, can account for the errors of discriminating between rotated symmetrical and asymmetrical stimuli at brief presentation durations. That the symmetry bias is perceptual in nature was evidenced by its refractoriness to manipulation by probability or familiarization. Massaro's (1973, 1975) extension-in-depth account of errors at high angles of orientation was not supported in that the large stimuli did not lead to a higher error rate than small stimuli as orientation angles increased.

\section{REFERENCES}

Brederman, I., \& Stacy, E. W., JR. Stimulus probability and set size in memory scanning. Journal of Experimental Psychology, 1974, 102. 1100-1107.

Biederman. I., \& Zachary, R. Stimulus versus response probability effects in choice reaction time. Perception \& Psychophysics, 1970, 7, 189-192.

Epstein, W., \& Park, J. N. Shape constancy: Functional relationships and theoretical formulations. Psychological Bulletin. 1963, 60. 265-288.

KoffKa, K. Principles of Gestalt psychology. New York: Harcourt. Brace, 1935.

Massaro. D. W. The perception of rotated shapes: A process analysis of shape constancy. Perception \& Psychophysics, 1973, 13, 413-422.

MASSARO, D. W, Experimental psychology and information processing. Chicago: Rand McNally, 1975.

OGLE. K. N. Researches in binocular vision. Philadelphia: Saunders. 1950

Shepard, R. N. Attention and the metric structure of the stimulus space. Journal of Mathematical Psychology, 1964, 1, 54-87.

Stravrianos. B. K. The relation of shape perception of explicit judgments of inclination. Archives of Psychology, 1945, 41. No. 296.

Thouless, R. H. Phenomenal regression to the real object. British Journal of Psychology, 1931, 21, 339-359.

\section{NOTES}

1. Fusion, the process where the separate images from each eye are combined into one cyclopean image becomes increasingly difficult and finally impossible as distance from the horopter increases. The horopter is defined as the locus of points in visual space giving rise to zero retinal disparity. The area of fusion is approximate; objects slightly off the horopter by $6-10 \mathrm{~min}$ of visual angle, Panum's area of single vision, will still fuse. However, beyond this area. objects are seen as blurry or double (Ogle, 1950). As a figure is rotated away from the frontal parallel plane (FPP) where the plane of the object is directly perpendicular to the viewer's line of sight. more of its surface area lies off the horopter circle. If the surface falls outside of Panum's area-out of the area of single vision-less of the figure will be fused during any one fixation. Thus, to determine the real shape of the figure, more vergence movements are necessary to sufficiently fuse enough of the figure to determine its shape.

2. Massaro (1975, p. 238) has offered his fusion model as an alternative to the shape-slant invariance hypothesis. Even if the fusion model was correct. it could not supplant shape-slant invariance because it does not specify an alternative relationship between perceived shape and perceived slant. Actually, all the fusion model does is to identify one possible source of increased RT and error with rotation.

3. Stimulus dimensions and presentation distances of our study were selected to match those employed by Massaro. However, if fixated at the axis of rotation. the far edge of the widest figures $(5.1 \mathrm{~cm})$ at the most extreme $\left(65^{\circ}\right)$ angles of rotation would yield too little disparity $\left(7^{\prime} 59^{\prime \prime}\right)$ for one to be confident that the disparity exceeded the $6^{\prime}-10^{\prime}$ of visual angle of Panum's area. However, since a fixation point was not used, fixations were probably only rarely dead center and, consequently, some of the figures on some of the trials might have fallen beyond Panum's area. So, while we can't make any precise statement as to the degree of disparity as a function of stimulus size, it is the case that the larger the figure, the more likely a noncentered fixation would result in sufficient disparity to exceed Panum's area.

4. Width-to-height ratios were used instead of height-to-width ratios to avoid the very large numbers that would result with the latter ratio when rotation is around the vertical axis and, therefore, projected width approaches zero. Height-to-width ratio measures would also yield converging functions (at infinity) as the angle of rotation approaches $90^{\circ}$.

(Received for publication March 17, 1975; revision accepted October $30,1975$. 